\title{
Bradykinin 1 receptor blockade subdues systemic autoimmunity, renal inflammation, and blood pressure in murine lupus nephritis
}

Ling Qin ${ }^{1,2}$, Yong Du ${ }^{2}$ Huihua Ding ${ }^{2}$, Anam Haque ${ }^{2}$, John Hicks ${ }^{3}$, Claudia Pedroza ${ }^{4}$ and Chandra Mohan ${ }^{2,1^{*}}$

\begin{abstract}
Objective: The goal of this study was to explore the role of bradykinins and bradykinin 1 receptor (B1R) in murine lupus nephritis.

Methods: C57BL/6 and MRL/pr mice were compared for renal expression of B1R and B2R by western blot and immunohistochemistry. MRL/Ipr lupus-prone mice were administered the B1R antagonist, SSR240612 for 12 weeks, and monitored for blood pressure, proteinuria, renal function, and serum autoantibodies.

Results: Renal B1R:B2R ratios were significantly upregulated in MRL/lpr mice compared with B6 controls. B1R blockade ameliorated renal pathology lesions, proteinuria, and blood pressure, accompanied by lower serum lgG and anti-dsDNA autoantibody levels, reduced splenic marginal zone B cells and CD4 ${ }^{+} \mathrm{T}$ cells, and renal infiltrating $\mathrm{CD}^{+} \mathrm{T}$ cells, macrophages, and neutrophils. Both urine and renal CCL2 and CCL5 chemokines were also decreased in the B1R blocked MRL/lpr mice.

Conclusion: Bradykinin receptor B1R blockade ameliorates both systemic immunity and renal inflammation possibly by inhibiting multiple chemokines and renal immune cell infiltration. B1R blockade may be particularly attractive in subjects with concomitant lupus nephritis and hypertension.
\end{abstract}

Keywords: Bradykinins, Hypertension, Lupus nephritis, Kallikrein

\section{Background}

Systemic lupus erythematosus (SLE) is a chronic systemic autoimmune disorder characterized by the production of autoantibodies, multiple organ involvement, and diverse clinical manifestations. Lupus nephritis (LN) is one of the commonest and most severe clinical features of SLE and leads to significant morbidity and mortality. Although 5- and 10-year SLE/LN survival rates have improved in recent years, significant challenges exist in understanding the pathogenesis of LN and designing appropriate therapy.

\footnotetext{
* Correspondence: cmohan@central.uh.edu

${ }^{2}$ Department of Biomedical Engineering, University of Houston, 3605 Cullen Boulevard, Houston, TX 77204, USA

'Department of Nephrology \& Rheumatology, Shanghai Tenth People's

Hospital, Tongji University School of Medicine, Shanghai, People's Republic of China

Full list of author information is available at the end of the article
}

Kinins are generated from kininogens catalyzed by kallikreins. Kinins exert their biological functions through two types of bradykinin receptors: B1R and B2R. B1R is expressed at inflammatory sites, whereas the $B 2 R$ is expressed in healthy tissue constitutively. It has been reported that B1R is involved in inflammation, pain, and fibrosis induced by inflammatory mediators. Indeed, this receptor-mediated pathway has been implicated in inflammatory bowel disease, vasculitis, experimentally induced nephritis, and acute gout [1-4]. In resonance with these reports, it has been shown that B1R antagonism or ablation plays a protective role in nephrotoxic serum-induced glomerulonephritis [5], lipopolysaccharide (LPS)-mediated acute renal inflammation [6], and experimental obstructive nephropathy [7]. However, the renal expression of B1R and its exact role in the pathogenesis of LN are poorly investigated. This is particularly

(C) The Author(s). 2019 Open Access This article is distributed under the terms of the Creative Commons Attribution 4.0 International License (http://creativecommons.org/licenses/by/4.0/), which permits unrestricted use, distribution, and 
important given that bradykinins are elevated in SLE [8]. This study was designed to explore the effect of B1R blockade on murine LN and to understand the underlying mechanisms.

\section{Materials and methods Mice and B1R blockade}

Female C57BL/6 (B6) and MRL/lpr mice were purchased from the Jackson Laboratory (Bar Harbor, ME, USA) and maintained in a specific pathogen-free colony. Animal experiments were approved and conducted in accordance with University of Houston's Institutional Animal Care regulations. Twenty-eight 4-month-old $\mathrm{MRL} / \mathrm{lpr}$ mice were divided into a control group ( $n=$ $14)$ and a treatment group $(\mathrm{n}=14)$ randomly for the in vivo studies. The B1R antagonist SSR240612 was purchased from Adooq Bioscience (Irvine, CA, USA). SSR240612 was dissolved in water containing dimethyl sulfoxide (DMSO) to make a final concentration of 1.5 $\mathrm{mg} / \mathrm{mL}$ in $0.9 \%$ DMSO. Mice in the treatment group were administered $10 \mathrm{mg} / \mathrm{kg}$ per day SSR240612 by gavage every other day, whereas the mice in the control group received $10 \mathrm{mg} / \mathrm{kg}$ per day $0.9 \%$ DMSO by gavage every other day; 24-h urine was collected using metabolic cages from all mice. Blood and urine were collected at 0,8 , and 12 weeks after treatment to assess proteinuria, serum blood urea nitrogen (BUN), alanine aminotransferase (ALT), and aspartate aminotransferase (AST). At 12 weeks after treatment, all mice were euthanized by using a $\mathrm{CO}_{2}$ chamber and cervical dislocation.

\section{Blood pressure measurement}

Blood pressure (BP) was monitored before and after 12 weeks of treatment using a non-invasive mouse-rat BP monitor (CODA, Kent Scientific, Torrington, CT, USA). The mean artery pressure (MAP) was used to compare the $\mathrm{BP}$ in the two groups.

\section{Renal histopathology}

Renal tissue was prepared as $4-\mu \mathrm{m}$ sections followed by formalin-fixation, dehydration, and paraffin-embedding. Slides were stained with hematoxylin and eosin or periodic acid-Schiff (PAS). Pathological changes in glomeruli, tubules, or interstitial areas were examined in a blinded fashion by a pathologist. Glomerulonephritis severity was graded on a $0-4$ scale in 20 glomeruli as follows: 0 , normal; 1 , mild increase in mesangial cellularity and matrix; 2 , moderate increase in mesangial cellularity and matrix, with thickening of the glomerular basement membrane (GBM); 3, focal endocapillary hypercellularity with obliteration of capillary lumina and a substantial increase in the thickness and irregularity of the GBM; 4, diffuse endocapillary hypercellularity, segmental necrosis, crescents, and hyalinized end-stage glomeruli. The interstitial score was determined by examining 20 high-power fields, and interstitial inflammation was scored on a scale from 0 to 4 as follows: 0 , no lesions; 1 , mild focal dilation or few foci of tubular atrophy or both; 2 , larger numbers of dilated tubules with widening of interstitium or larger numbers of foci of tubular atrophy or both; 3 , extensive dilation of tubules with cyst formation and widening of interstitium or a large numbers of foci of tubular atrophy or both; 4, extensive tubular atrophy [9]. Glomeruli with any degree of sclerosis or collapse and thrombonecrotic lesions were graded on a $0-4$ scale, corresponding to absence of lesions, or involving less than $10 \%, 11-20 \%, 21-30 \%$, or more than $31 \%$ of glomeruli, respectively.

\section{Immunohistochemistry}

Five B6 mice and five MRL/lpr mice at 4 months of age were used to assess renal expression of B1R and B2R. Kidney sections obtained from 4-month-old C57BL/6 J and MRL/lpr mice were stained with the following primary antibodies: rabbit anti-mouse B1R antibody (Bioss Inc., Woburn, MA, USA) and rabbit anti-mouse B2R antibody (Bioss Inc.). Antigen retrieval was performed by using a sodium citrate buffer $(10 \mathrm{mM}$ sodium citrate, $\mathrm{pH}$ 6.0) in a microwave oven, protein-blocked for 20 $\mathrm{min}$, and endogenous enzyme-blocked for $20 \mathrm{~min}$, followed by incubation with dextran polymer conjugated with horseradish peroxidase (HRP) and affinity-isolated immunoglobulins, using diaminobenzidine $(\mathrm{DAB})+$ as chromagen. All reagents were purchased from Dako (Santa Clara, CA, USA).

\section{Western blot}

Western blot was performed as described previously [10]. In brief, total renal protein was extracted and prepared in sample buffer by boiling for $10 \mathrm{~min}$. Samples were spun down, subjected to SDS-PAGE, and transferred to a PVDF membrane using a Bio-Rad Trans-Blot Turbo transfer system. Rabbit anti-mouse B1R antibody (Bioss Inc.), rabbit anti-mouse B2R antibody (Bioss Inc.), and anti- $\alpha$-tubulin (Cell Signaling Technology, Beverly, MA, USA) were used as the primary antibodies. HRP-conjugated secondary antibodies and the ECL-plus detection kit (Amersham, Little Chalfont, UK) were used for western blot. For analysis, bands were quantified by Imagej".

\section{Flow cytometry}

Animals were sacrificed and the spleens and kidneys were collected for flow cytometry analysis. The monoclonal antibodies used for splenic flow cytometry were CD4-PE, CD3-PE-cy7, Foxp3-FITC, CD69-percp-cy7, B220-PE-cy7, CD21-FITC, CD23-PE, CD11c-PE-cy7, CD11b-APC, F4/80-PE, CD86-FITC, and F4/80-PerCP. The monoclonal antibodies used for renal flow 
cytometry were CD4-PE, CD3-Percp, Foxp3-FITC, CD45-APC-cy7, CD11b-FITC, CD11c-PE-cy7, F4/80-PE, and Gr-1-Percp (eBioscience, Hanover Park, IL, USA). Cell counting was performed by using a Cellometer automated cell counting system (Sigma-Aldrich, St Louis, MO, USA) for absolute cell numbers. The Novocyte flow cytometer system (ACEA Bioscience Inc., San Diego, CA, USA) was used for flow cytometry, and analysis was performed as described [11]. Data were analyzed using Novocyte software (ACEA Bioscience Inc.). At least 200,000 events were acquired for each analysis.

\section{Total serum IgG and autoantibody detection}

Total serum IgG was determined by using commercial enzyme-linked immunosorbent assay (ELISA) kits in accordance with the instructions of the manufacturer (eBioscience, San Diego, CA, USA). In brief, anti-mouse IgG was first coated onto plates and blocked. Test samples were diluted serially and added to the plates for $2 \mathrm{~h}$ at room temperature, followed by incubation with detection antibody, substrate solution, and stop solution. Concentrations were determined by using a standard curve.

ELISA assay for anti-dsDNA, ssDNA, and histone autoantibodies was performed as described previously [12]. Briefly, Immulon $2 \mathrm{~B}$ plates were pre-treated with $1 \%$ mBSA in phosphate-buffered saline, and $50 \mu \mathrm{g} / \mathrm{mL}$ dsDNA or ssDNA (or histone) was added, and blocked, before adding a 1:400 dilution of mouse serum. All autoantibodies were detected with HRP-conjugated goat anti-mouse IgG, and the plates were read at $405 \mathrm{~nm}$. All reagents were obtained from Sigma-Aldrich. Pooled serum from lupus-afflicted MRL/lpr mice with a starting dilution of $1 / 100$ served as the standard. These absorbance values were fit to derive a curve, using a four-parameter fit, and all samples' absorbance values were converted to arbitrary units by using this standard curve.

\section{ELISA for urine or kidney lysate chemokines}

In this study, urine and kidney lysate CCL2, CCL5, and CXCL9 levels were measured by using ELISA kits from R\&D Systems (Minneapolis, MN, USA) in accordance with the instructions of the manufacturer. Briefly, diluted urine or kidney lysate samples were added to capture antibody pre-coated 96-well microplates. After incubation with samples, the detection antibody was added, followed by streptavidin-HRP, and substrate. A microplate reader ELX808 from BioTek Instruments (Winooski, VT, USA) was used to read the optical density at $450 \mathrm{~nm}$. The concentration was calculated based on a standard curve. Urinary creatinine concentrations were determined by using Creatinine Parameter Assay Kit (R\&D Systems). Urine creatinine concentrations were used to normalize urine chemokine concentrations.

\section{Statistics}

Data were analyzed and plotted by using GraphPad Prism 5 software (GraphPad Software, San Diego, CA, USA). The Kolmogorov-Smirnov test was used to assess the normality of the data. For comparison between two groups, the $t$ test was used when the normality test passed; otherwise, a non-parametric Mann-Whitney test was used to analyze the data. A two-tailed $P$ value of less than 0.05 was considered significant. For all data, the statistical results were also re-computed after correcting for the missing data due to the deceased animals (by assigning to the deceased mice the mean values recorded for the respective phenotypes in the surviving mice in the treatment group or control group). These corrected $P$ values are appended to the legends of Figs. 3, 4, and 5 .

\section{Results}

Renal expression of B1R and B2R in MRL/Ipr mice assayed using two complementary approaches

To explore the renal expression of bradykinin receptors B1R and B2R in MRL/lpr mice, total renal protein was extracted and examined by western blot. Renal B1R expression was increased in MRL/lpr mice compared with C57BL/6 J mice (Fig. 1a, c), whereas renal B2R expression was decreased in MRL/lpr mice compared with C57BL/6 J mice (Fig. 1b, d). All mice were 4 months old at the time of examination. Immunohistochemistry analysis was also used to validate renal B1R and B2R expression. Immunohistochemistry did not reveal renal B1R expression in C57BL/6 J mice (Fig. 2a), whereas B1R-positive staining was detected in the glomeruli and renal tubules of $\mathrm{MRL} / \mathrm{lpr}$ mice (Fig. 2b). In contrast, B2R-positive staining was detected in glomeruli in $\mathrm{C} 57 \mathrm{BL} / 6 \mathrm{~J}$ mice (Fig. 2c); renal expression of B2R was decreased in $\mathrm{MRL} / \mathrm{lpr}$ mice compared with the controls (Fig. 2d), consistent with the western blot results.

\section{The effect of bradykinin receptor B1R blockade on body weight and spleen and kidney weight in MRL/lpr mice} B1R blockade did not affect the body weight of MRL/lpr mice (Additional file 1: Figure S1). No significant difference was seen in the ratio of spleen weight to body weight between the control group and the treatment group (Fig. 3a). In addition, it was found that B1R blockade decreased the ratio of kidney weight to body weight compared with the control group (Fig. 3a, $P=0.0409$ ).

\section{Bradykinin receptor B1R blockade reduced blood pressure in MRL/lpr mice}

Kinins are involved in BP regulation. To ascertain whether B1R blockade can impact BP in MRL/lpr mice, we monitored the BP before and after B1R antagonist treatment (Fig. 3b). Baseline MAP BP levels were similar 

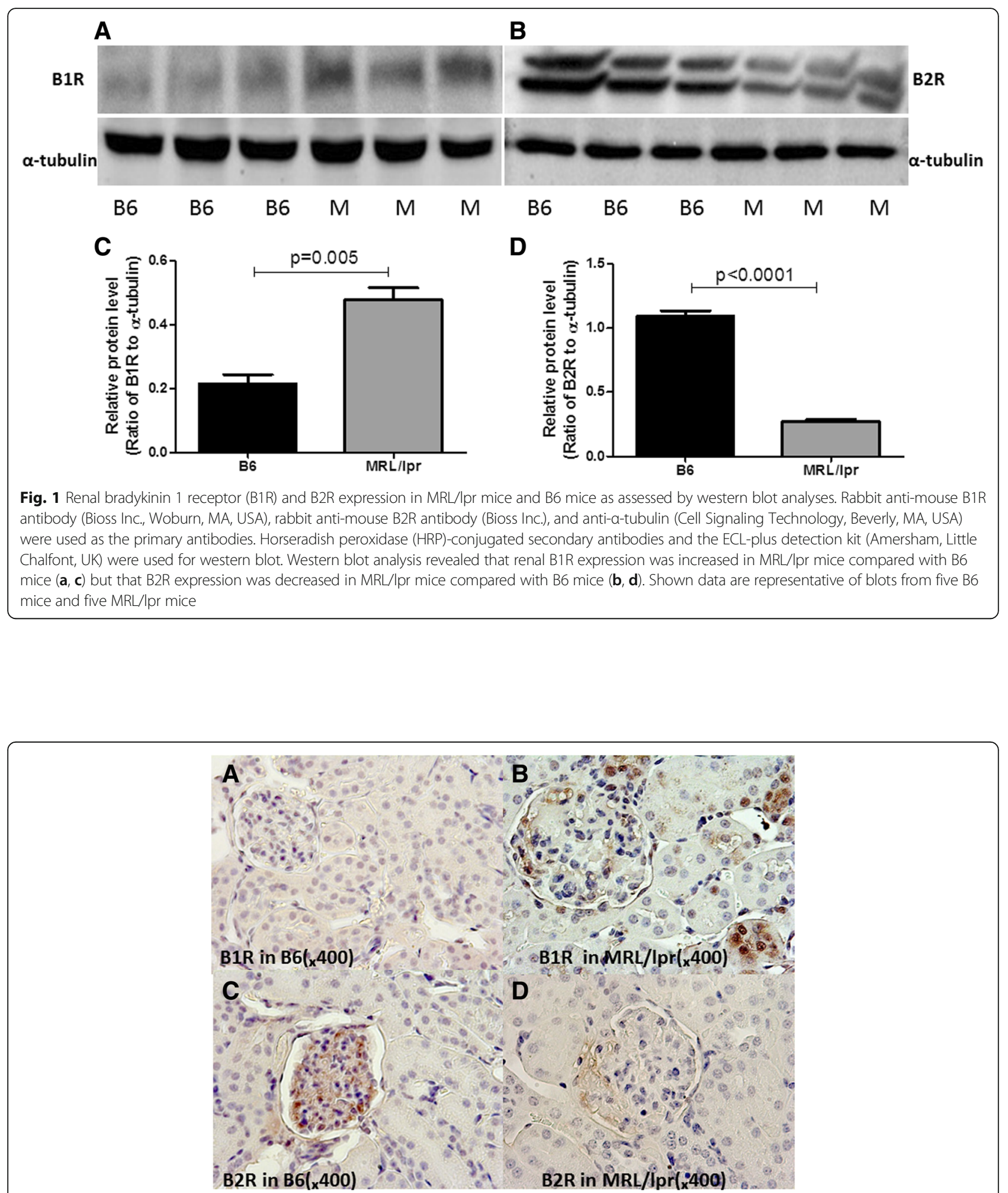

Fig. 2 Renal bradykinin 1 receptor (B1R) and B2R expression in MRL/lpr mice and B6 mice as assessed by immunohistochemistry (IHC). IHC was used to monitor renal B1R and B2R expression in control and lupus mice. Kidney sections obtained from 4-month-old C57BL/6 J and MRL/lpr mice were stained with the following primary antibodies: rabbit anti-mouse B1R antibody (Bioss Inc. Woburn, MA, USA) and rabbit anti-mouse B2R antibody (Bioss Inc. Woburn, MA, USA). IHC analysis indicated elevated renal B1R expression in MRL/lpr mice (b) compared with B6 mice (a). B6 mice demonstrated stronger renal B2R expression than MRL/lpr mice (c, d). Shown data are representative of IHC staining from five B6 mice and five MRL/lpr mice 


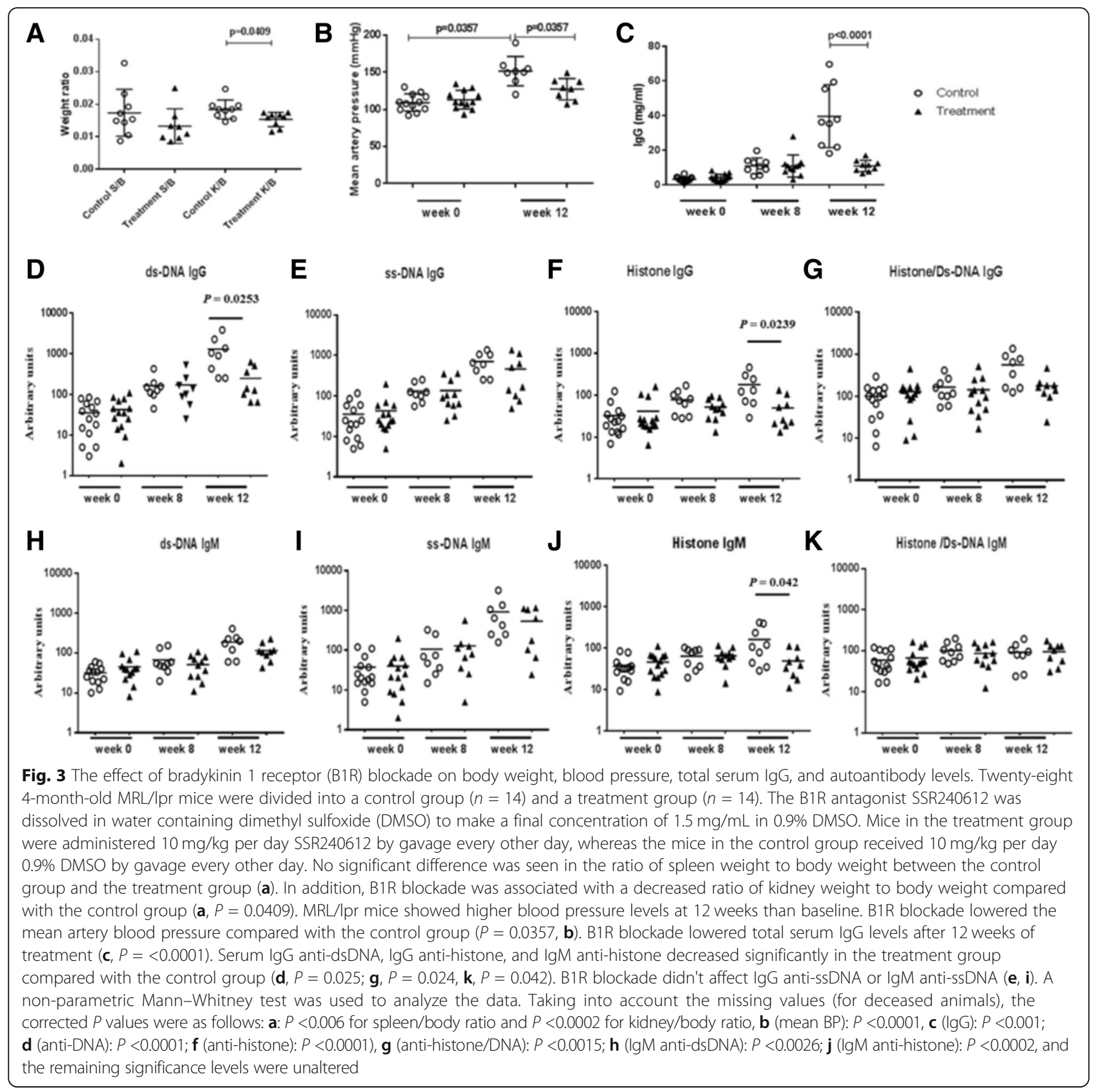

in the two groups of mice $(104.3 \pm 14.29 \mathrm{~mm} \mathrm{Hg}$ versus $109.0 \pm 21.85 \mathrm{~mm} \mathrm{Hg}$ ). The MAP of the control group of MRL/lpr mice was $155.8 \pm 20.59 \mathrm{~mm} \mathrm{Hg}$ at 12 weeks. B1R blockade lowered the MAP in treated MRL/lpr mice to $122.5 \pm 10.00 \mathrm{~mm} \mathrm{Hg}$, which is significantly lower compared with the control group.

\section{Bradykinin receptor B1R blockade reduced circulating levels of autoantibodies and IgG}

To assess the effect of B1R blockade on systemic immune response in MRL/lpr mice, we assayed total serum IgG levels and autoantibody levels after the
B1R blockade. We found that B1R blockade lowered total serum IgG concentrations after 12 weeks of treatment (Fig. 3c, $P<0.0001$ ) but not total IgM (data not shown). IgG anti-dsDNA, IgG anti-Histone, and IgM anti-Histone antibodies were also decreased significantly in the treated mice compared with the control group (Fig. 3d, $P=0.025$; Fig. $3 g, P=0.024$, Fig. $3 k, P=0.042)$. However, the reduction in most of the other autoantibodies assayed attained statistical significance once the data were corrected for the missing values for the deceased animals at the 12-week time point (Fig. 3). 


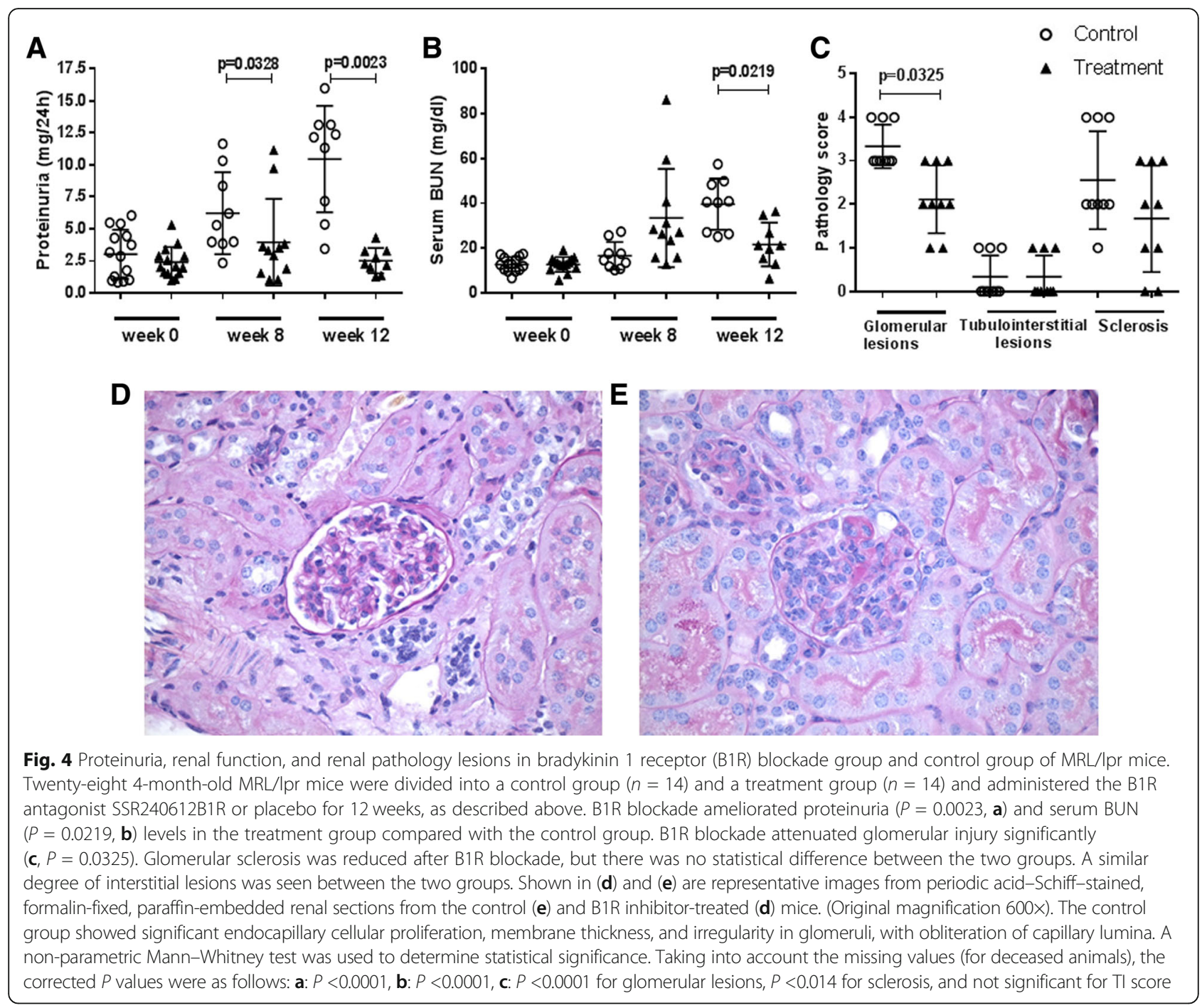

\section{Bradykinin receptor B1R blockade reduced proteinuria and serum BUN in MRL/lpr mice}

In view of the increased renal expression of $B 1 R$ in murine $\mathrm{LN}$, we next investigated the effect of B1R blockade on proteinuria and renal function. B1R blockade diminished proteinuria 8 weeks after treatment (Fig. 4a, $P=0.0328$ ). Both proteinuria and serum BUN were lower after 12 weeks of treatment compared with the control group (Fig. 4a, b, $P=0.0023, P=0.0219$ ), indicating that B1R blockade subdues LN.

\section{Bradykinin receptor B1R blockade regulated systemic and local intra-renal immune cell populations}

To investigate possible mechanisms through which B1R blockade may be mitigating $\mathrm{LN}$, splenocytes and intra-renal cells were isolated from the control group and the treatment group, and flow cytometry was used to interrogate the immune cell populations (Table 1). Total splenocytes were decreased after 12 weeks of B1R antagonist treatment. Absolute cell numbers of $\mathrm{CD}^{+} \mathrm{CD} 4^{+}, \mathrm{B} 220^{+}$, and $\mathrm{B} 220^{+} \mathrm{CD} 21^{\text {high }} \mathrm{CD} 23^{\text {low }}$ marginal zone $\mathrm{B}$ cells in spleens were also decreased in the treated mice compared with the control group. No significant difference was seen in total intra-renal immune cells between the two groups. It was also observed that the absolute cell numbers of intra-renal $\mathrm{CD} 45^{+} \mathrm{CD}^{+} \mathrm{CD}^{+}, \mathrm{CD} 45^{+} \mathrm{CD} 11 \mathrm{~b}^{+} \mathrm{F} 4 / 80^{+}$, and $\mathrm{CD} 45^{+} \mathrm{CD} 11 \mathrm{~b}^{+} \mathrm{Gr}-1^{+}$infiltrating cells were lowered after 12 weeks of B1R blockade compared with the control MRL/lpr mice (Table 1).

\section{Bradykinin receptor B1R blockade prevented progression of renal disease}

We next investigated the therapeutic effect of B1R blockade on renal lesions. Untreated MRL/lpr mice showed progressive renal injury (Fig. 4e). In contrast, treatment with the B1R antagonist ameliorated glomerular injury significantly (Fig. 4c, d, $P=0.0325$ ). No difference in interstitial lesions was observed between the two 
Table 1 Immune cells subsets in MRL/lpr mice

\begin{tabular}{|c|c|c|}
\hline & $\begin{array}{l}\text { MRL/lpr } \\
\text { control mice }\end{array}$ & MRL/Ipr + SSR treatment \\
\hline \multicolumn{3}{|l|}{ Spleen: absolute cell numbers $\left(\times 10^{6}\right)$} \\
\hline Total cells & $174.1 \pm 16.8$ & $88.90 \pm 17.06^{* *}$ \\
\hline Total T cells $\left(\mathrm{CD}^{+}\right)$ & $63.15 \pm 9.14$ & $40.75 \pm 19.97$ \\
\hline $\mathrm{CD}^{+} \mathrm{CD}^{+}$cells & $9.51 \pm 1.13$ & $5.04 \pm 0.81^{* *}$ \\
\hline $\mathrm{CD}^{+} \mathrm{CD}^{+}$cells & $2.67 \pm 0.75$ & $1.56 \pm 0.61$ \\
\hline $\mathrm{CD}^{+} \mathrm{CD}^{+} \mathrm{CD} 9^{+}$cells & $2.56 \pm 1.28$ & $1.76 \pm 0.21$ \\
\hline $\mathrm{CD}^{+} \mathrm{CD}^{+}$foxp3 cells & $0.97 \pm 0.21$ & $0.59 \pm 0.32$ \\
\hline Total B cells $\left(B 220^{+}\right)$ & $104.5 \pm 25.82$ & $47.85 \pm 16.62^{*}$ \\
\hline $\mathrm{B} 220^{+} \mathrm{CD} 21^{\text {high }} \mathrm{CD} 23^{\text {low }}$ cells & $11.82 \pm 1.86$ & $5.94 \pm 1.29^{* *}$ \\
\hline $\mathrm{B} 220^{+} \mathrm{CD} 23^{\text {high }} \mathrm{CD} 21^{\text {low }}$ cells & $6.48 \pm 1.85$ & $10.34 \pm 6.31$ \\
\hline $\mathrm{CD} 11 \mathrm{~b}^{+} \mathrm{F} 4 / 80^{+} \mathrm{CD} 86^{+}$cells & $1.80 \pm 0.23$ & $1.10 \pm 0.74$ \\
\hline $\mathrm{CD}_{11 \mathrm{c}^{+}} \mathrm{CD} 6^{+}$cells & $9.72 \pm 1.65$ & $6.54 \pm 2.50$ \\
\hline \multicolumn{3}{|l|}{ Kidney: absolute cell numbers $\left(\times 10^{5}\right)$} \\
\hline Total cells & $27.16 \pm 5.73$ & $18.97 \pm 1.22$ \\
\hline Total infiltrating cells $\left(\mathrm{CD} 45^{+}\right)$ & $6.92 \pm 1.63$ & $4.75 \pm 1.61$ \\
\hline $\mathrm{CD}_{4} 5^{+} \mathrm{CD}^{+} \mathrm{CD}^{+}$cells & $1.57 \pm 0.29$ & $0.91 \pm 0.18^{*}$ \\
\hline $\mathrm{CD}_{4} 5^{+} \mathrm{CD}^{+} \mathrm{CD}^{+}$foxp3 cells & $0.18 \pm 0.05$ & $0.17 \pm 0.04$ \\
\hline $\mathrm{CD}_{4} 5^{+} \mathrm{CD} 11 \mathrm{~b}^{+} \mathrm{F} 4 / 80^{+}$cells & $3.06 \pm 1.27$ & $1.04 \pm 0.12^{*}$ \\
\hline $\mathrm{CD}_{4} 5^{+} \mathrm{CD} 11 \mathrm{c}^{+}$cells & $3.52 \pm 2.07$ & $1.96 \pm 0.94$ \\
\hline $\mathrm{CD}_{4} 5^{+} \mathrm{CD} 11 \mathrm{~b}^{+} \mathrm{Gr}-1^{+}$cells & $0.43 \pm 0.07$ & $0.20 \pm 0.006^{*}$ \\
\hline
\end{tabular}

${ }^{*} P<0.05 ;{ }^{* * P}<0.01$

groups of mice. Once the data were corrected for the missing data due to the deceased animals, the reduction in glomerulosclerosis scores also attained statistical significance (Fig. 4 legend). We next examined whether the reduced disease in the treated mice had an impact on survival. The survival times after treatment were $68 \pm$ 21 days in the control group and $76 \pm 14$ days in treatment group (log-rank test $P=0.087$ ). Thus, B1R blockade did not significantly increase the survival time of the lupus mice (Additional file 1: Figure S3).

\section{Bradykinin receptor B1R blockade reduced urine and renal chemokine expression}

To ascertain the effect of B1R blockade on renal chemokine expression, we used ELISA to assay levels of urine CCL2, CCL5, and CXCL9, chemokines reported to be elevated in MRL/lpr lupus mice. Compared with the healthy counterparts, urine CCL2 and CCL5 were increased in MRL/lpr mice; however, B1R blockade decreased urine CCL2 and CCL5 levels after 12 weeks of treatment (Fig. 5a, $P=0.0076$; Fig. 5b, $P=0.0339$ ). B1R blockade did not affect urine CXCL9 levels (Fig. 5c). To explore intra-renal chemokine expression, we measured renal lysate CCL2 and CCL5 levels by ELISA. Renal CCL2 and CCL5 levels were elevated in MRL/lpr mice compared with B6 mice (Fig. 5d, $P=0.0011$; Fig. 5e, $P=0.0135$ ).
B1R blockade significantly decreased renal CCL2 and CCL5 levels compared with those of the control MRL/lpr mice (Fig. 5d, $P=0.0319$; Fig. 5e, $P=0.0299$ ) and these reductions became even more significant once the data were corrected for the missing values due to the deceased mice (Fig. 5 legend).

\section{Screening for potential side effects of therapy}

As described earlier, B1R blockade did not significantly impact the body weight of the treated mice (Additional file 1: Figure S1). To monitor the effect of B1R blockade on liver function, serum ALT and AST were measured. The ALT and AST levels were similar between the control group and treatment group at 0 and 12 weeks (Additional file 1: Figure S2A and S2B).

\section{Discussion}

Kallikreins catalyze the production of kinins from kininogens. Kallikreins and kinins exert multiple biological functions, including the regulation of cytokine release, pain, edema, leukocyte recruitment, and cell proliferation [13]. In our previous studies, we have reported that kallikrein gene polymorphisms are associated with lupus and nephrotoxic serum-induced nephritis $[14,15]$. In addition, kallikreins play a reno-protective role in LN and nephrotoxic serum-induced nephritis [16]. However, 


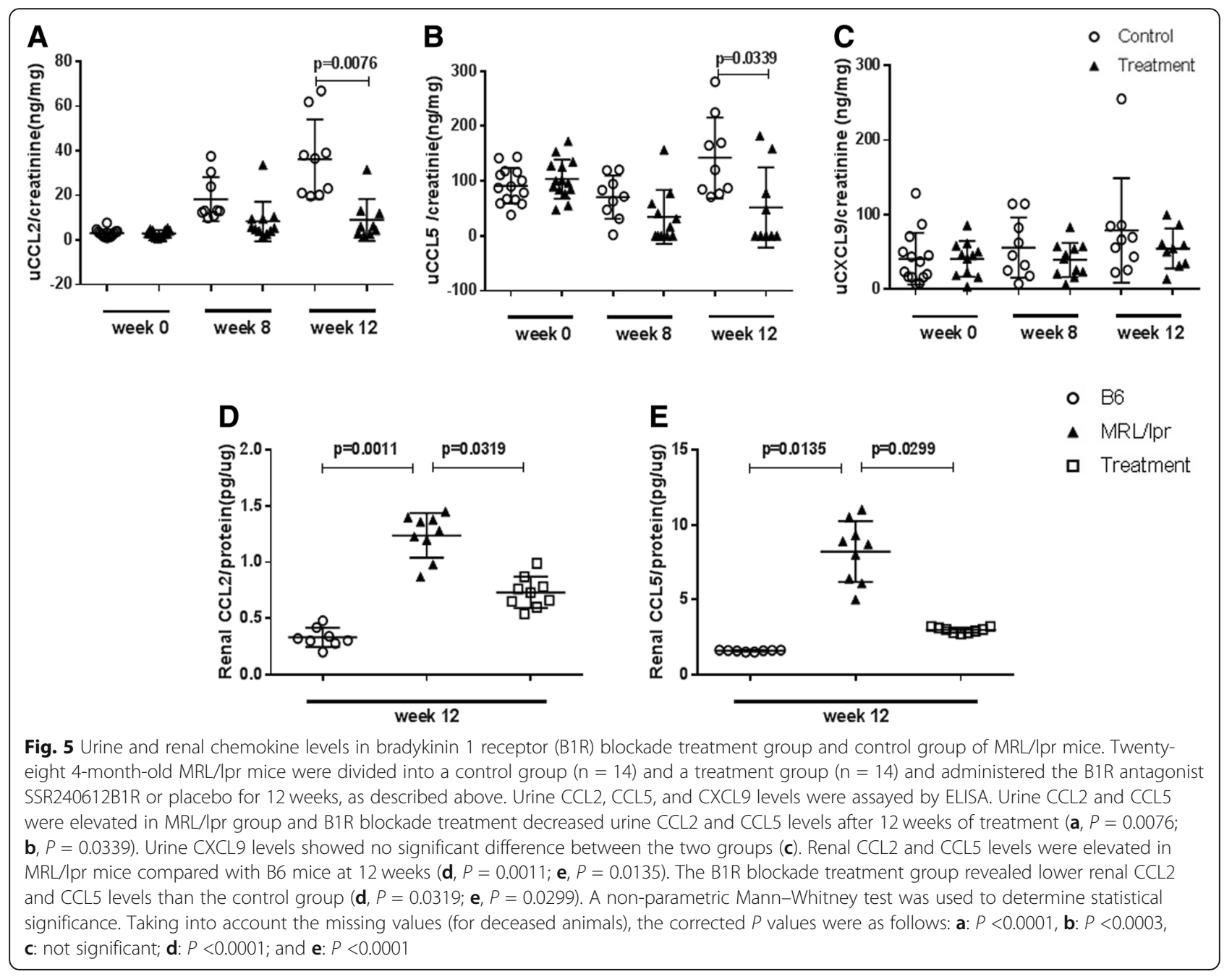

the molecular mechanisms for this reno-protection have not been systematically investigated.

It is known that kallikreins promote the generation of bradykinins, BK and BK-des-Arg9. Whereas BK binds to the B2R bradykinin receptor, BK-des-Arg9 binds to and activates another bradykinin receptor, B1R, particularly under inflammatory conditions [17]. B1R is expressed at inflammatory sites, induced by inflammatory mediators, such as interleukin-1 (IL-1) and tumor necrosis factor-alpha (TNF- $\alpha$ ), in a nuclear factor-kappa B (NF-kB)- and mitogen-activated protein kinase (MAPK)-dependent manner $[18,19]$. In particular, the B1R appears to play a key role in inflammation, pain, and fibrosis and has been implicated in inflammatory bowel disease, multiple sclerosis, and experimentally induced nephritis $[1,5,20,21]$. Moreover, the B1R is expressed along the nephron and is involved in renal inflammation and fibrosis in other renal disease models [5, 22].

Along the same line, Pereira et al. [3] reported that B1R agonist exacerbated experimental focal and segmental glomerulonephritis (FSGS) but that B1R antagonist reduced proteinuria and glomerulofibrosis, reversed podocyte dysfunction, and played a protective role in the pathogenesis of FSGS. It has been documented that B1R staining was positive in renal tissue of patients with antineutrophil cytoplasmic antibody (ANCA)-associated vasculitis and Henoch-Schönlein purpura nephritis [5]. B1R blockade or ablation was also documented to be effective in ameliorating renal fibrosis in experimental obstructive nephropathy [7], reducing the renal inflammatory response in cisplatin or LPS-induced acute renal injury and ischemic-reperfusion injury in murine models $[6,23,24]$. In our previous work in anti-GBM-induced experimental nephritis mice, blockade of the B2R bradykinin receptor worsened disease whereas blockade of B1R ameliorated disease [15], once again suggesting the pro-inflammatory and pathogenic role of the B1R in immune-mediated nephritis. Considering those previous studies, we also speculated that B2R may mediate a reno-protective effect once engaged by $\mathrm{BK}$ in autoimmune nephritis. The goal of this study is to extend these findings to $\mathrm{LN}$. 
Our study demonstrated that renal B1R expression was increased but that B2R expression was decreased in MRL/ lpr lupus-prone mice. More importantly, renal B1R:B2R ratios were significantly increased and this could significantly skew the effects of kinins to be mediated primarily via pro-inflammatory pathways. Interestingly, no literature has been reported on end-organ B1R:B2R ratios in any disease model. Given the accumulating evidence that B1R may be pro-inflammatory and B2R may be renoprotective, renal B1R:B2 ratios may be an important determinant of renal inflammation, possibly with diagnostic or disease-predictive potential. Our study also demonstrates that disease amelioration in MRL/lpr mice may be associated with reduced intra-renal chemokine expression and immune infiltrates.

A number of chemokines have been shown to play important roles in LN. Renal CCL2, CCL5, and CXCL9 mRNA and protein have been reported to be elevated in MRL/lpr mice and the roles of these chemokines and their corresponding receptors have been documented in murine LN [25-27]. It has been speculated that B1R may contribute to disease by regulating inflammatory cytokines, such as CCL2, MIP-1, and CCL5, in experimental FSGS [3]. SSR240612 was initially reported as a novel non-peptide antagonist of the B1R with selectivity for $B 1 R$ versus $B 2 R$ in the range of 500 - to 1000 -fold, where SSR240612 inhibited BK-des-Arg9 induced inositol monophosphate formation in human fibroblast MRC5 with a half maximal inhibitory concentration $\left(\mathrm{IC}_{50}\right)$ of $1.9 \mathrm{nM}$ [28]. Subsequently, SSR240612 has been successfully used as a B1R antagonist in animal models of other renal and non-renal diseases $[5,29,30]$. B1R blockade has also been reported to reduce renal inflammation by downregulating renal CCL2, CCL5, and CCL7 in the anti-GBM nephritis model [5]. In addition, renal CCL2 and CCL7 overexpression was observed in an obstructive nephropathy model and B1R antagonist was demonstrated to inhibit renal inflammation and fibrosis in vivo and in vitro, partially mediated by inhibiting CCL2 and CCL7 expression [25]. Consistent with the earlier reports, B1R blockade in murine $\mathrm{LN}$ is also associated with downregulation of renal chemokines, notably CCL2 and CCL5, in the present study. Consistent with the chemokine reduction within the kidneys, B1R blockade in MRL/lpr mice also reduced intra-renal levels of $\mathrm{CD}_{4} 5^{+} \mathrm{CD} 3^{+} \mathrm{CD} 4^{+}$ $\mathrm{T}$ cells, $\mathrm{CD} 45^{+} \mathrm{CD} 11 \mathrm{~b}^{+} \mathrm{F} 4 / 80^{+}$macrophages, and $\mathrm{CD} 45^{+}$ $\mathrm{CD} 11 \mathrm{~b}^{+} \mathrm{Gr}-1^{+}$granulocytes, possibly driven in part by alterations in CCL2 and CCL5 expression [31].

To our surprise, B1R blockade not only subdued LN but also suppressed systemic autoimmunity, as noted by the reduction in total serum IgG and anti-dsDNA IgG levels, as well as splenic marginal zone $\mathrm{B}$ cells and $\mathrm{CD} 4^{+}$ $\mathrm{T}$ cells. It has been reported that B1R expression was upregulated on $\mathrm{T}$ cells from peripheral blood of patients with multiple sclerosis [20, 32]. Moreover, B1R expression has been reported on dendritic cells [33]. Although B1R expression on systemic immune cells in LN was not examined in this study, our results support the hypothesis that bradykinin receptor blockade may serve to ameliorate systemic immunity by silencing dendritic cells and helper $\mathrm{T}$ cells, which in itself should be sufficient to ameliorate lupus, both at the systemic level and the end-organ disease manifestations, including LN.

Our study also revealed that B1R blockade reduced BP in $\mathrm{MRL} / \mathrm{lpr}$ mice. Previously, it has been reported that B1R blockade exerts a BP-lowering effect in hypertensive rat models [29, 34]. It has also been reported that brain B1R antagonist decreased BP via a raclopride sensitive mechanism by downregulation of dopaminergic pathways which otherwise may have hypertensive effects [29]. Additionally, it has been reported that activation of B1R elevates superoxide anions by activating NADPH oxidase in the vasculature; moreover, B1R antagonist treatment was shown to decrease high BP in 12-week glucose-fed rats by reducing oxidative stress [30]. It has been shown that excess of either superoxide or hydrogen peroxide in the renal medulla reduces renal medullary blood flow and enhances $\mathrm{Na}^{+}$reabsorption and hypertension [35]. B1R blockade in the present study may have reduced BP in MRL/lpr mice via several different mechanisms. Indeed, other mechanisms not related to the bradykinin pathway may also have indirectly contributed to the reduction in BP but this warrants further investigation. Although hypertension is not a feature of lupus autoimmunity, a substantial fraction of patients with SLE and LN also have hypertension as a comorbidity. Thus, the anti-hypertensive effect of B1R blockade constitutes an additional beneficial impact of this therapeutic modality in LN.

Despite the improvement in autoimmunity, LN, and hypertension, there was no significant difference in survival following B1R blockade. However, the observation that treated mice survived 8 days longer on average $(P<0.087)$ warrants a repeat of these studies with a larger animal cohort or longer treatment duration or both. In addition, the potential impact of increased drug dosage needs to be examined.

\section{Conclusions}

In summary, these studies have yielded several novel observations. First, there is a dramatic imbalance in bradykinin receptor expression within LN kidneys, and significantly heightened B1R:B2R receptor ratios resulted in increased levels of the pro-inflammatory B1R. Second, these studies show that targeting the bradykinin B1Rs may offer therapeutic benefit in three different ways: (a) amelioration of systemic lupus and dampening of systemic autoimmunity and autoantibodies, (b) reduced 
LN, possibly mediated by lowering of chemokines and intra-renal immune infiltrates, and (c) reducing the BP. Further studies are clearly warranted to explore the utility of bradykinin B1R blockade in patients with SLE given the multiple avenues through which this axis impacts disease.

\section{Additional file}

Additional file 1: Figure S1. Body weight in the control group and treatment group of MRL/lpr mice. Figure S2. Liver function in the control group and treatment group of MRL/lpr mice. Figure S3. Impact of bradykinin 1 receptor (B1R) blockade on mortality in MRL/lpr mice. (DOCX $83 \mathrm{~kb})$

\section{Abbreviations}

ALT: Alanine aminotransferase; AST: Aspartate aminotransferase; B1R: Bradykinin 1 receptor; BP: Blood pressure; BUN: Blood urea nitrogen; DMSO: Dimethyl sulfoxide; ELISA: Enzyme-linked immunosorbent assay; FSGS: Focal and segmental glomerulonephritis; GBM: Glomerular basement membrane; HRP: Horseradish peroxidase; LN: Lupus nephritis; LPS: Lipopolysaccharide; MAP: Mean artery pressure; SLE: Systemic lupus erythematosus

\section{Acknowledgments}

We acknowledge the technical assistance of Xiang Li, Research Assistant Professor of Pharmacology, Department of Pharmacological and Pharmaceutical Sciences, University of Houston (Houston, TX).

\section{Funding}

These studies were supported by funding from the Alliance for Lupus Research and the National Natural Science Foundation of China (grant 81571574). The funding bodies did not participate in or influence the design of the study; collection, analysis, and interpretation of data; or writing the manuscript.

\section{Availability of data and materials}

Please contact CM for data requests.

\section{Authors' contributions}

$L Q, Y D, H D, A H$, and $J H$ carried out various experiments and generated the data. $L Q, Y D, A H$, and $C M$ wrote different parts of the manuscript. $L Q, C P$, and CM analyzed the data. CM conceived the study, participated in its design and coordination, and helped to draft the manuscript. All authors read and approved the final manuscript.

\section{Ethics approval and consent to participate}

Animal experiments were approved by and conducted in accordance with the University of Houston's Institutional Animal Care regulations.

\section{Consent for publication}

Not applicable.

\section{Competing interests}

The authors declare that they have no competing interests.

\section{Publisher's Note}

Springer Nature remains neutral with regard to jurisdictional claims in published maps and institutional affiliations.

\section{Author details}

'Department of Nephrology \& Rheumatology, Shanghai Tenth People's Hospital, Tongji University School of Medicine, Shanghai, People's Republic of China. ${ }^{2}$ Department of Biomedical Engineering, University of Houston, 3605 Cullen Boulevard, Houston, TX 77204, USA. ${ }^{3}$ Texas Children's Hospital, Houston, TX, USA. ${ }^{4}$ University of Texas at Houston, Houston, TX, USA.
Received: 26 February 2018 Accepted: 25 November 2018

Published online: 08 January 2019

\section{References}

1. Marceau F, Regoli D. Therapeutic options in inflammatory bowel disease: experimental evidence of a beneficial effect of kinin B1 receptor blockade. Br J Pharmacol. 2008:154:1163-5.

2. Kahn R, Mossberg M, Stahl AL, Johansson K, Lopatko Lindman I, Heijl C, et al. Microvesicle transfer of kinin B1-receptors is a novel inflammatory mechanism in vasculitis. Kidney Int. 2017;91:96-105.

3. Pereira RL, Buscariollo BN, Correa-Costa M, Semedo P, Oliveira CD, Reis VO, et al. Bradykinin receptor 1 activation exacerbates experimental focal and segmental glomerulosclerosis. Kidney Int. 2011;79:1217-27.

4. Silva CR, Oliveira SM, Hoffmeister C, Funck V, Guerra GP, Trevisan G, et al. The role of kinin B1 receptor and the effect of angiotensin I-converting enzyme inhibition on acute gout attacks in rodents. Ann Rheum Dis. 2016;75:260-8.

5. Klein J, Gonzalez J, Decramer S, Bandin F, Neau E, Salant DJ, et al. Blockade of the kinin B1 receptor ameloriates glomerulonephritis. J Am Soc Nephrol. 2010;21:1157-64.

6. Bascands JL, Bachvarova M, Neau E, Schanstra JP, Bachvarov D. Molecular determinants of LPS-induced acute renal inflammation: Implication of the kinin B1 receptor. Biochem Biophys Res Commun. 2009:386:407-12.

7. Huart A, Klein J, Gonzalez J, Buffin-Meyer B, Neau E, Delage C, et al. Kinin B1 receptor antagonism is equally efficient as angiotensin receptor 1 antagonism in reducing renal fibrosis in experimental obstructive nephropathy, but is not additive. Front Pharmacol. 2015;6:8

8. Wu T, Xie C, Han J, Ye Y, Weiel J, Li Q, et al. Metabolic disturbances associated with systemic lupus erythematosus. PLoS One. 2012;7:e37210.

9. Xie C, Zhou XJ, Liu X, Mohan C. Enhanced susceptibility to end-organ disease in the lupus-facilitating NZW mouse strain. Arthritis Rheum. 2003:48:1080-92.

10. Wu T, Ye Y, Min SY, Zhu J, Khobahy E, Zhou J, et al. Prevention of murine lupus nephritis by targeting multiple signaling axes and oxidative stress using a synthetic triterpenoid. Arthritis Rheumatol. 2014;66:3129-39.

11. Wang A, Guilpain P, Chong BF, Chouzenoux S, Guillevin L, Du Y, et al. Dysregulated expression of CXCR4/CXCL12 in subsets of patients with systemic lupus erythematosus. Arthritis Rheum. 2010;62:3436-46.

12. Li L, Nukala S, Du Y, Han J, Liu K, Hutcheson J, et al. Murine lupus strains differentially model unique facets of human lupus serology. Clin Exp Immunol. 2012;168:178-85.

13. Ponticelli C, Meroni PL. Kallikreins and lupus nephritis. J Clin Invest. 2009; 119:768-71.

14. Li QZ, Zhou J, Yang R, Yan M, Ye Q, Liu K, et al. The lupus-susceptibility gene kallikrein downmodulates antibody-mediated glomerulonephritis. Genes Immun. 2009;10:503-8.

15. Liu K, Li QZ, Delgado-Vega AM, Abelson AK, Sanchez E, Kelly JA, et al. Kallikrein genes are associated with lupus and glomerular basement membrane-specific antibody-induced nephritis in mice and humans. J Clin Invest 2009:119:911-23.

16. Li Y, Raman I, Du Y, Yan M, Min S, Yang J, et al. Kallikrein transduced mesenchymal stem cells protect against anti-GBM disease and lupus nephritis by ameliorating inflammation and oxidative stress. PLoS One. 2013;8:e67790.

17. Bockmann S, Paegelow I. Kinins and kinin receptors: importance for the activation of leukocytes. J Leukoc Biol. 2000;68:587-92.

18. Cunha TM, Verri WA Jr, Fukada SY, Guerrero AT, Santodomingo-Garzon T, Poole $\mathrm{S}$, et al. TNF-alpha and IL-1 beta mediate inflammatory hypernociception in mice triggered by B1 but not B2 kinin receptor. Eur J Pharmacol. 2007;573:221-9.

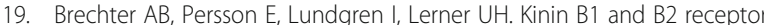
expression in osteoblasts and fibroblasts is enhanced by interleukin-1 and tumour necrosis factor-alpha. Effects dependent on activation of NF-kappaB and MAP kinases. Bone. 2008:43:72-83.

20. Prat A, Weinrib L, Becher B, Poirier J, Duquette P, Couture R, et al. Bradykinin B1 receptor expression and function on $T$ lymphocytes in active multiple sclerosis. Neurology. 1999:53:2087-92

21. Shao X, Yang R, Yan M, Li Y, Du Y, Raman I, et al. Inducible expression of kallikrein in renal tubular cells protects mice against spontaneous lupus nephritis. Arthritis Rheum. 2013:65:780-91.

22. Klein J, Gonzalez J, Duchene J, Esposito L, Pradere JP, Neau E, et al. Delayed blockade of the kinin B1 receptor reduces renal inflammation and fibrosis in obstructive nephropathy. FASEB J. 2009;23:134-42. 
23. Estrela GR, Wasinski F, Almeida DC, Amano MT, Castoldi A, Dias CC, et al. Kinin $\mathrm{B} 1$ receptor deficiency attenuates cisplatin-induced acute kidney injury by modulating immune cell migration. J Mol Med (Berl). 2014;92:399-409.

24. Wang PH, Campanholle G, Cenedeze MA, Feitoza CQ, Goncalves GM, Landgraf RG, et al. Bradykinin [corrected] B1 receptor antagonism is beneficial in renal ischemia-reperfusion injury. PLoS One. 2008;3:e3050,

25. Perez de Lema G, Maier H, Nieto E, Vielhauer V, Luckow B, Mampaso F, et al. Chemokine expression precedes inflammatory cell infiltration and chemokine receptor and cytokine expression during the initiation of murine lupus nephritis. J Am Soc Nephrol. 2001;12:1369-82.

26. Turner JE, Paust HJ, Bennstein SB, Bramke P, Krebs C, Steinmetz OM, et al. Protective role for CCR5 in murine lupus nephritis. Am J Physiol Renal Physiol. 2012;302:F1503-15.

27. Steinmetz OM, Turner JE, Paust HJ, Lindner M, Peters A, Heiss K, et al. CXCR3 mediates renal Th1 and Th17 immune response in murine lupus nephritis. J Immunol. 2009;183:4693-704.

28. Gougat J. SSR240612 [(2R)-2-[((3R)-3-(1,3-Benzodioxol-5-yl)-3-ÂA propanoyl)amino]-3-(4-Â phenyl)-N-isopropyl-N-methylpropanamide Hydrochloride], a New Nonpeptide Antagonist of the Bradykinin B1 Receptor:Biochemical and Pharmacological Characterization. J Pharmacol Exp Ther. 2004;309(2):661-69

29. De Brito Gariepy H, Carayon P, Ferrari B, Couture R. Contribution of the central dopaminergic system in the anti-hypertensive effect of kinin B1 receptor antagonists in two rat models of hypertension. Neuropeptides. 2010;44:191-8.

30. Dias JP, Talbot S, Senecal J, Carayon P, Couture R. Kinin B1 receptor enhances the oxidative stress in a rat model of insulin resistance: outcome in hypertension, allodynia and metabolic complications. PLoS One. 2010;5:e12622.

31. Haberstroh U, Pocock J, Gomez-Guerrero C, Helmchen U, Hamann A, Gutierrez-Ramos JC, et al. Expression of the chemokines MCP-1/CCL2 and RANTES/CCL5 is differentially regulated by infiltrating inflammatory cells. Kidney Int. 2002;62:1264-76.

32. Gobel K, Pankratz S, Schneider-Hohendorf T, Bittner S, Schuhmann MK, Langer $\mathrm{HF}$, et al. Blockade of the kinin receptor B1 protects from autoimmune CNS disease by reducing leukocyte trafficking. J Autoimmun. 2011;36:106-14.

33. Bertram CM, Baltic S, Misso NL, Bhoola KD, Foster PS, Thompson PJ, et al. Expression of kinin B1 and B2 receptors in immature, monocyte-derived dendritic cells and bradykinin-mediated increase in intracellular $\mathrm{Ca} 2+$ and cell migration. J Leukoc Biol. 2007:81:1445-54.

34. Emanueli C, Chao J, Regoli D, Chao L, Ni A, Madeddu P. The bradykinin B1 receptor and the central regulation of blood pressure in spontaneously hypertensive rats. Br J Pharmacol. 1999;126:1769-76.

35. Cowley AW Jr, Abe M, Mori T, O'Connor PM, Ohsaki Y, Zheleznova NN. Reactive oxygen species as important determinants of medullary flow, sodium excretion, and hypertension. Am J Physiol Renal Physiol. 2015;308:F179-97.

Ready to submit your research? Choose BMC and benefit from:

- fast, convenient online submission

- thorough peer review by experienced researchers in your field

- rapid publication on acceptance

- support for research data, including large and complex data types

- gold Open Access which fosters wider collaboration and increased citations

- maximum visibility for your research: over $100 \mathrm{M}$ website views per year

At $\mathrm{BMC}$, research is always in progress.

Learn more biomedcentral.com/submissions 